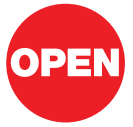

SUBJECT AREAS:

COMPUTATIONAL

CHEMISTRY

CONDENSED MATTER PHYSICS

MODELLING AND THEORY

NANOTECHNOLOGY

Received

10 March 2011

Accepted

23 June 2011

Published

13 July 2011

Correspondence and requests for materials should be addressed to

P.K. (pkral@uic.edu)

\section{Electronic structures of porous nanocarbons}

\author{
Artem Baskin \& Petr Král
}

Department of Chemistry, University of Illinois at Chicago, Chicago, IL 60607, USA.

We use large scale ab-initio calculations to describe electronic structures of graphene, graphene nanoribbons, and carbon nanotubes periodically perforated with nanopores. We disclose common features of these systems and develop a unified picture that permits us to analytically predict and systematically characterize metal-semiconductor transitions in nanocarbons with superlattices of nanopores of different sizes and types. These novel materials with highly tunable band structures have numerous potential applications in electronics, light detection, and molecular sensing.

\begin{abstract}
7 raphene ${ }^{1}$ has unique and highly tunable parameters, which can be exploited in novel hybrid materials and devices with numerous applications. It can be modified by doping ${ }^{2,3}$, chemical functionalization ${ }^{4}$, and geometrical restrictions, such as cutting and introduction of defects and pores ${ }^{5-7}$. Recently, graphene perforated with nanopores was used as a selective sieve for hydrated ions ${ }^{11}$, gases ${ }^{12,13}$, and DNA ${ }^{15,16}$.

In this work, we use large scale ab-initio calculations to describe electronic structures of nanocarbons perforated with superlattices of nanopores. We search for common principles allowing us to characterize the electronic structures of porous nanocarbons (PNC), such as porous graphene (PG), porous graphene nanoribbons (PGNR), and porous carbon nanotubes (PCNT). Although, partial results for the electronic structures of $\mathrm{PG}^{5-7}$ and other PNCs have been obtained ${ }^{17-20}$, general principles that would unify their electronic structures are missing.
\end{abstract}

\section{Results}

The studied PNCs are perforated with pores of different shapes, sizes, and locations. Most of the results are obtained for arrays of honeycomb-shaped pores, called the "standard pore" (SP $)^{13,14}$, where in each pore six C atoms (one benzene ring) are excluded from the studied nanocarbon and the dangling bonds are terminated by hydrogens. All the PNCs have their edges and pores passivated by hydrogen atoms. The details of our calculations are described in Methods.

Porous graphene nanoribbons. We begin our study by examining the band structures of porous armchair (AGNR) and zigzag (ZGNR) graphene nanoribbons. All GNRs are classified by the number of carbon dimers, $N$, that form the ribbon $(N-G N R)^{20}$. First, we study the porous 11-AGNR and 10-ZGNR (both metallic when pristine), and elementary cells are shown in Figs. 1 (d, h). In Figs. 1 (a, b), we can see that the introduction of a periodic array of SPs in the center of 11-AGNR causes a significant band-gap opening $(0.15 \mathrm{eV} \rightarrow 0.92 \mathrm{eV})$. On the other hand, both pristine and porous (same pores) 10-ZGNR have no energy band gap, as seen in Figs. 1 (e, f). When the SPs are displaced by one honeycomb cell towards the GNR-edge, the band gap in 11-AGNR shrinks $\approx$ 3.75 times, while 10-ZGNR remains metallic, as shown in Figs. 1 (c, g). We also checked that the PGNR band structures monotonously approach their pristine form as the separation between adjacent pores is increased. The densities of states (DOS) for the band structures presented are displayed in Figs. 1 (d, h).

The energy band gaps in ZGNRs and AGNRs are known to arise from a staggered sublattice potential and a quantum confinement ${ }^{21,22}$, respectively, and they depend on the ribbon width and its functionalization ${ }^{4,23,24}$. The wave functions of the HOMO and LUMO bands in AGNRs, which contribute directly to the area near $E_{f}$, are localized at the center of the ribbons, keeping their edges chemically stable ${ }^{4}$. In ZGNRs, these wavefunctions are localized at the ribbon edges. Consequently, when the SPs are positioned at the center of 11-AGNR a band gap opens in its band structure, while 10-ZGNR remains metallic. The metallicity of ZGNRs is caused by flat bands present at $E_{f}$, originating from highly localized states formed at the zigzag edges ${ }^{4}$, as discussed below. When the pore is closer to the edge of 11-AGNR, its band structure is influenced less, while no significant change is observed in 10-ZGNR. 

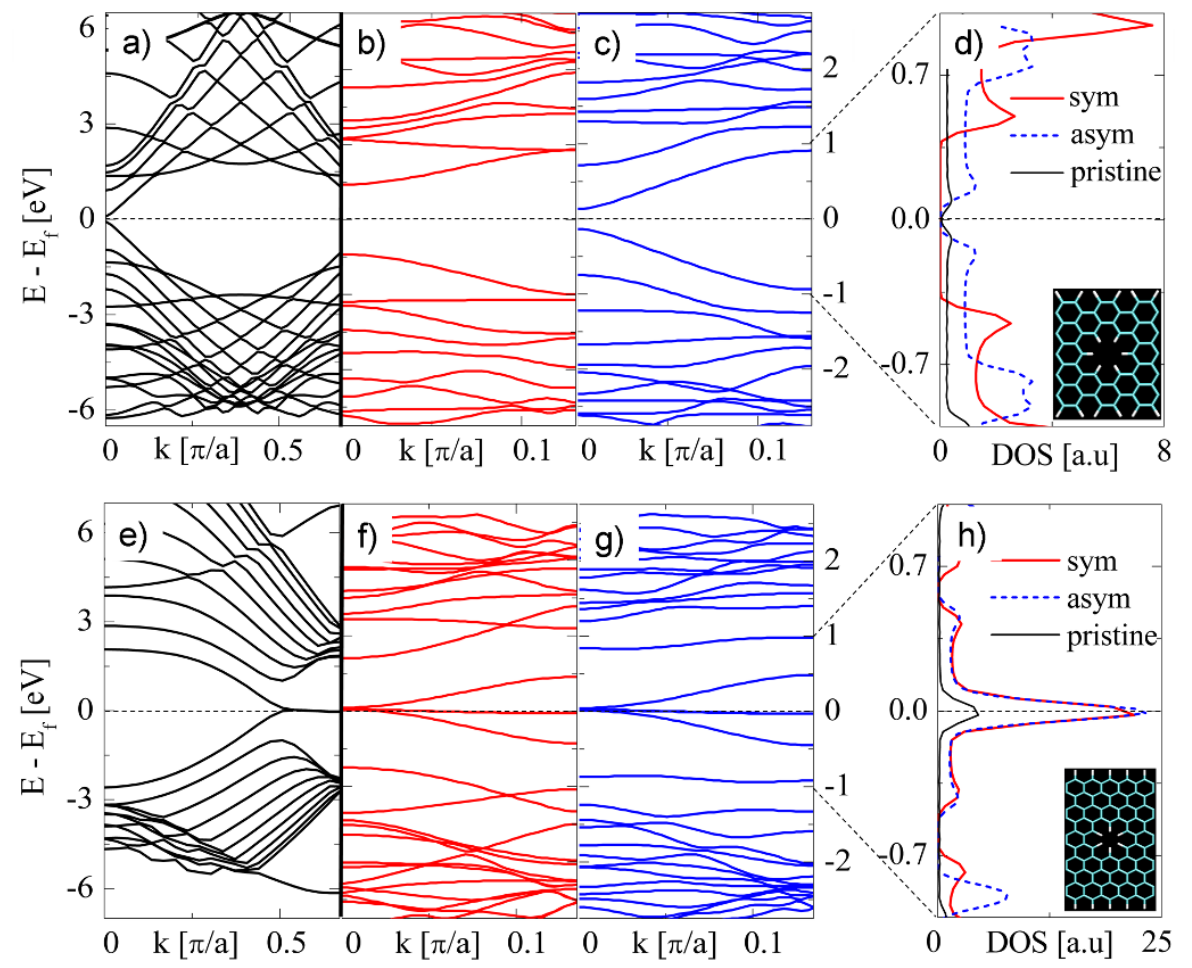

Figure 1 Band structure of: a) pristine 11-AGNR, b) 11-AGNR with centered SP, c) 11-AGNR with shifted SP, e) pristine 10-ZGNR, f) 10-ZGNR with centered SP, g) 10-ZGNR with shifted SP. The energy scales for (b, c, f, g) cases are the same. Density of states: d) centered and shifted SP in 11-AGNR, h) centered and shifted SP in 10-ZGNR. (insets) Unit cells for 11-AGNR and 10-ZGNR with the standard pore ( $a$ is the lattice constant of the supercell).

Porous graphene. We continue to study PG that lacks explicit edges, in contrast to PGNRs. Graphene-based systems have a bipartite lattice ${ }^{20}$, with $n_{A}$ and $n_{B}$ sites per unit cell in the $A$ and $B$ sublattices, leading to $n_{A}-n_{B}$ flat bands at the Fermi level ${ }^{7}$. The metallicity caused by these bands is highly stable to any perturbation (see Figs. 1 (f, g)). Although, $n_{A}=n_{B}=1$ in graphene, introduction of pores or other defects may change its global sublattice balance, i.e. $n_{A} \neq n_{B}$. The relationship between edge-localized states, zigzag-like edges of GNR, and flat bands at the Fermi level was discussed in ${ }^{8}$. It turns out that even a small number of zigzag sites at the ribbon edge gives rise to highly localized states forming flat bands at $E_{f}$. These zigzag edges are characterized by a local sublattice imbalance and unbalanced $\pi$-electron spin density ${ }^{9}$. These localized states at zigzag rims were also studied in different types of pores, called "antimolecules", where a set of simple rules was shown to link the net number of unpaired electrons with the degeneracy of flat bands $^{2}, 5,7,10,26$. SPs, largely used in our study, keep the global sublattice balance of the unit cell, but their short zigzag-like rims break the local balance and may still give rise to flat bands.

In Figs. $2(\mathrm{a}-\mathrm{d})$, we show the band structures of (edgeless) PGs with one honeycomb and three different rectangular SP-superlattices defined by $N_{A}, N_{Z}$, and displayed in Figs. $2(\mathrm{e}, \mathrm{f})^{25}$. Figure $2(\mathrm{~g})$ also shows the studied rectangular superlattice with triangular-shape pores. The honeycomb SP-superlattice has flat bands around $E \approx-1.5 \mathrm{eV}$ (Fig. 2 a), which are absent in the rectangular SPsuperlattices (Figs. 2 (b-d)). According to ${ }^{7}$, such quasi-flat bands at non-zero energy might be ascribed to the local sublattice imbalance (globally $n_{A}=n_{B}$ ). However, the local sublattice imbalance due to SPs can not be the origin of flat bands in the band structure of the honeycomb SP-superlattice, since it has the same pores as the other structures with no flat bands. Therefore, the flat bands are most likely related with the honeycomb SP-arrangement, which might produce differently localized states. These results show that the small number of zigzag sites at the very short SP-rim can not generate flat bands at the Fermi level or at its vicinity. In contrast, even the smallest triangular-shape pores break the global sublattice balance and generate an unbalanced $\pi$-electron density ${ }^{9}$, associated with the appearance of rim-localized states and giving rise to flat bands at $E_{f}$ (not shown).

With these observations, we can now relate the band structures of PGNRs and PGs. Since SPs do not break the global sublattice balance in the PGNRs, their presence does not generate any new flat bands at $E_{f}$ (see Fig. 1); on the contrary, we found that when AGNRs are perforated with triangular-shape pores, their band structure always contains flat bands at $E_{f}$. Even though the SPs do not create flat bands at $E_{f}$ in GNRs, they still influence their band structure. While the band structures of AGNRs (Figs. 1 (b, c)) is influenced a lot, the band structures of ZGNRs with flat bands at $E_{f}$ (local sublattice imbalance caused by zigzag edges) can not be significantly modified by SPs. Therefore, we conclude that all porous ZGNRs are metallic (checked by calculations).

We performed extensive $a b$ initio calculations of electronic structures of PG-superlattices with different arrangements of the SPs. Interestingly, we found that rectangular PG-superlattices perforated with SPs and larger pores of honeycomb symmetry can be both metallic and semiconducting. It turns out that we can generalize these observations into a hypothesis that, in the first approximation, the electronic structure of these superlattices has the same type of conductivity as many parallel AGNRs or ZGNRs (of effective widths $N_{A}$ or $N_{Z}$ ), depending on the ratio $\rho=N_{A} / N_{Z}$ (see Fig. 3 bottom). If $\rho \gg 1$, one can see the superlattice as being "cut" into separated $N_{A^{-}}$ AGNRs, while for $\rho \ll 1$ the same is true for separated $N_{Z}$-ZGNRs.

The above hypothesis was largely confirmed by our follow up calculations. For example, the conductivity in the PGs with $N_{Z}=2$ is the same as in the corresponding AGNRs: metallic for $N_{A}=$ $5,11,17 \ldots$ and semiconducting for $N_{A}=7,9,13,15 \ldots$ (see also Fig. 5). If we continue with the $N_{A}=9,15,21, \ldots$ semiconducting AGNRs and increase the initially small $N_{Z}=2$, we find that the PGs remain semiconducting for (roughly) $N_{Z}<N_{A}$, with the band gap shrinking with increasing $N_{Z}$, signaling the transition to the 

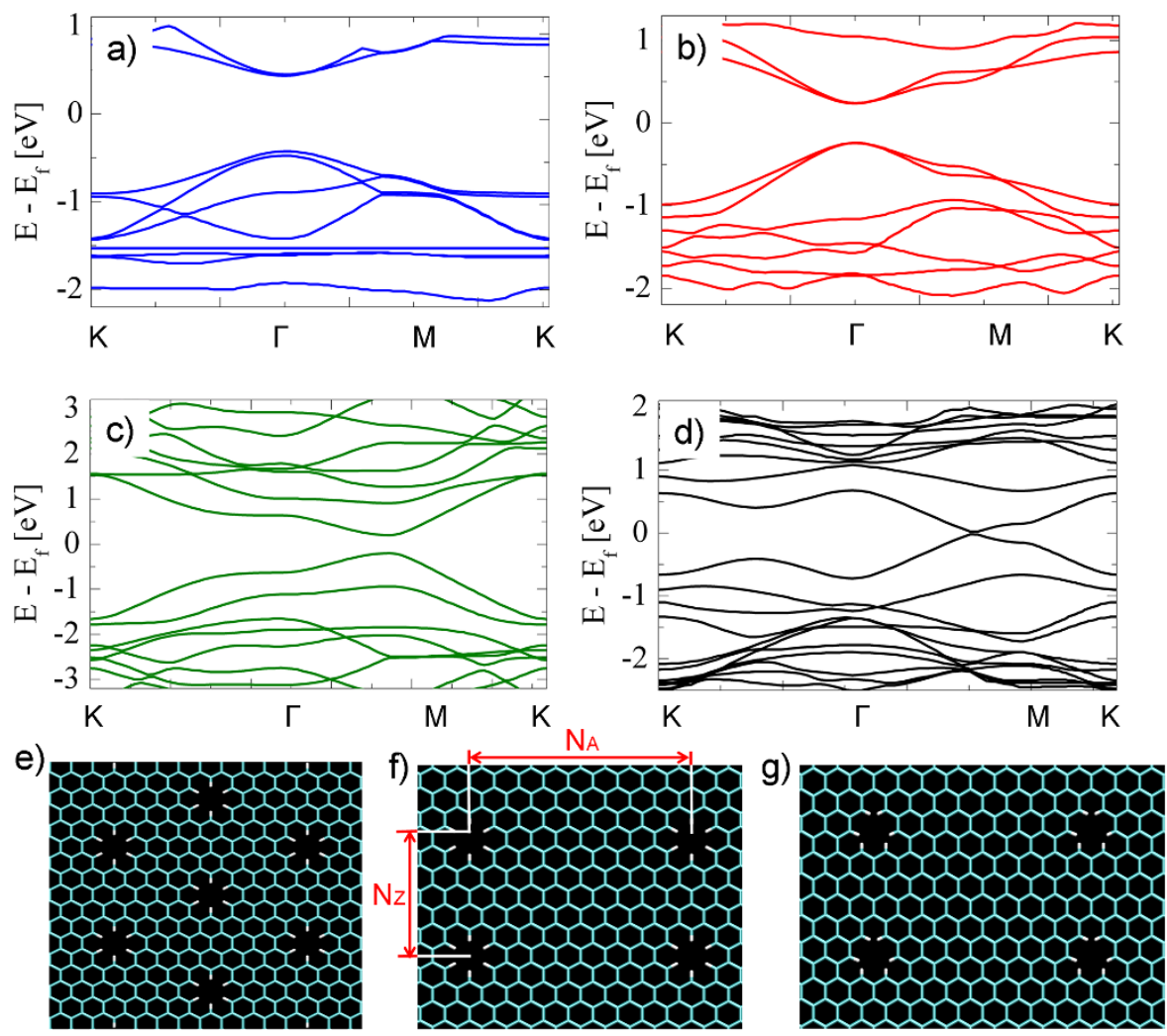

Figure $2 \mid$ (top) Band structure of a) a honeycomb SP-superlattice in e) and three rectangular SP-superlattices with b) $N_{A}=15, N_{Z}=4, \mathrm{c}$ ) $N_{A}=7, N_{Z}=$ 2, and d) $N_{A}=7, N_{Z}=8$. (bottom) e) honeycomb SP-superlattice, f) rectangular SP-superlattice characterized by $N_{A}, N_{Z}\left(\right.$ example with $N_{A}=15, N_{Z}=4$ is shown), g) rectangular superlattice with triangular-shape pores.

ZGNR-dominated metallic conductivity. If we continue with the the $N_{A}=11$ metallic AGNRs, the PGs become metallic for all the $N_{Z}$, since the ZGNRs that take over at $N_{Z}>N_{A}$ are all metallic. Finally, when we continue from $N_{A}=7,13 \ldots$, the metallicity appears abruptly at $N_{Z} \geq 4$. In other cases, we expect that the transition between the AGNR and ZGNR-type of behavior occurs somewhere around the "diagonal", $\rho=1$. Our calculations also show that the metal-semiconductor transitions predominantly occur in two regions of the Brillouin zones, as seen in Figs. $2(\mathrm{~b}-\mathrm{d})$, and the bands can be partially flat in the $k_{y}$ direction $\left(N_{A}=7,13, \ldots\right)$.

Porous nanotubes. It turns out that there is also a clear correspondence between pristine CNTs and structurally analogous GNRs (GNRs can be rolled into CNTs). While ZGNRs indexed by
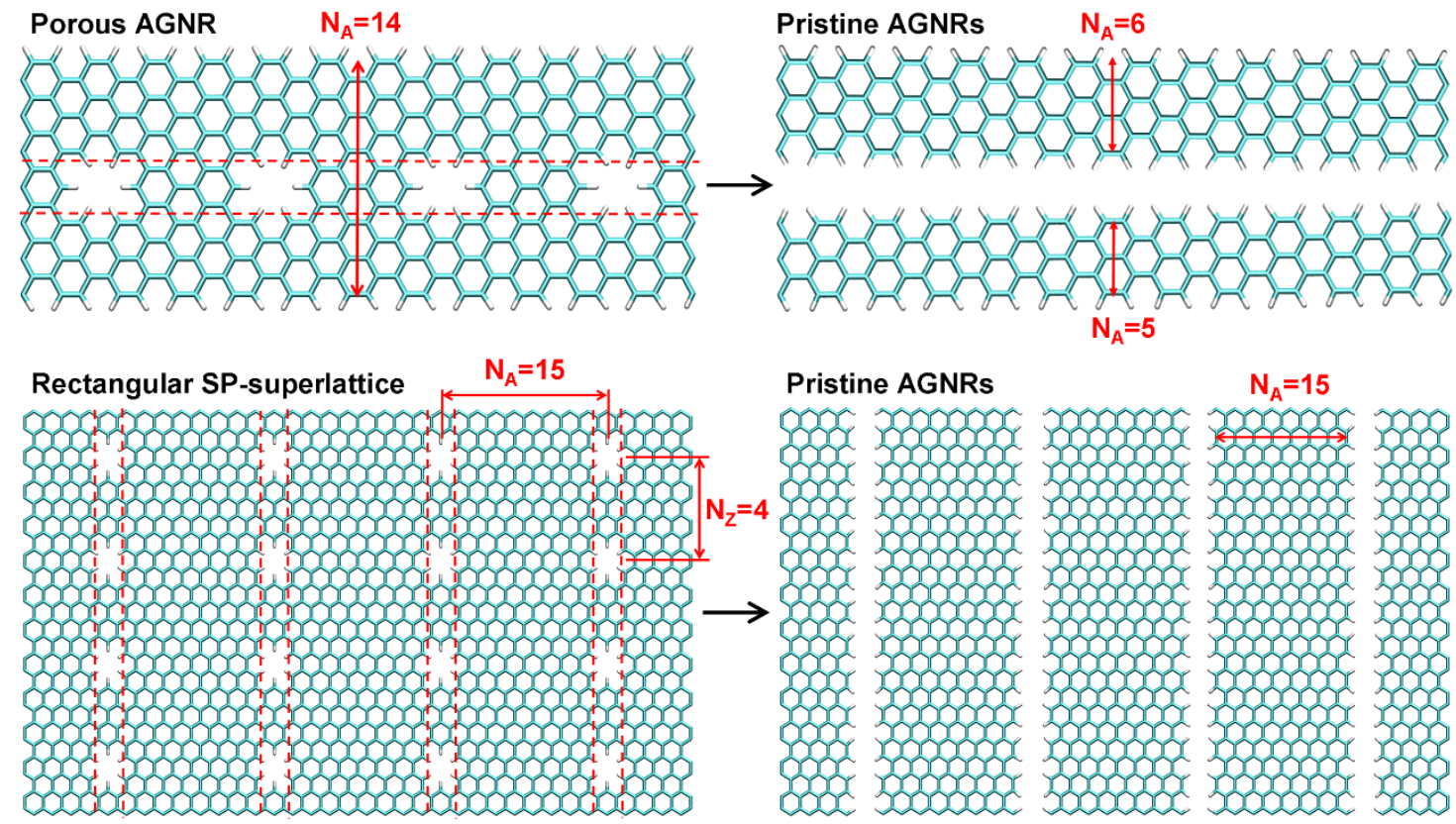

Figure $3 \mid$ (top) Effective replacing of porous N-AGNR by “daughter" pristine $N_{1^{-}}$and $N_{2}$-AGNR $\left(N=14, N_{1}=5, N_{2}=6\right)$. (bottom) Effective replacing of rectangular SP-superlattice with $N_{A}>N_{Z}$ by set of prestine AGNRs $\left(N_{A}=15, N_{Z}=4\right)$. 


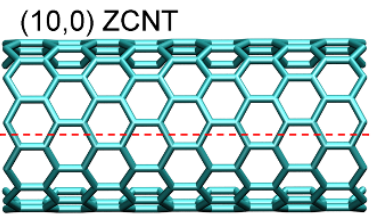

$(9,9)$ ACNT

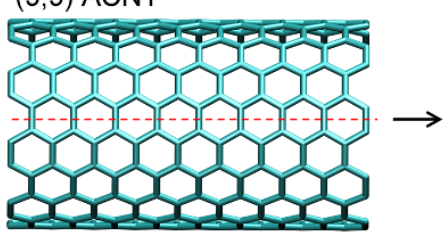

Porous $(10,10)$ ACNT

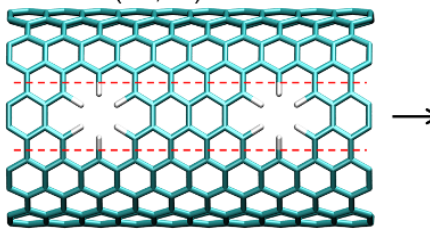

Figure $4 \mid$ Cutting of: (top) (10,0)ZCNT into $\langle 19,1\rangle$ AGNR and (middle) $(9,9) \mathrm{ACNT}$ into $\langle 18,0\rangle \mathrm{ZGNR}$ (opening of the GNRs is schematically shown). (bottom) Removal of atoms from porous $(10,10)$ ACNT leading to $\langle 17,0\rangle$ ZGNR.

$\langle p, 0\rangle$ are metallic for all $p$, only one third of AGNRs indexed by $\langle p, 1\rangle$ are metallic. Since, only one half of AGNRs can be rolled into ZCNTs, only one third ZCNTs are metallic ${ }^{27}$. We found that this mapping can be generalized to the point that every metallic/semiconducting CNT corresponds to a metallic/semiconducting GNR. The unambiguous relationship can be expressed in terms of the chirality index, $p$, and the number of GNRs carbon dimers, $N$, as follows

$$
\begin{array}{llll}
\mathrm{ZCNT}\langle p, 0\rangle & \Rightarrow N-\mathrm{AGNR}, & \text { wher } & N=2 p+2, \\
\operatorname{ACNT}\langle p, p\rangle \Rightarrow N-\mathrm{ZGNR}, & \text { wher } & N=2 p+1 .
\end{array}
$$

Intuitively, we can look at this CNT-GNR correspondence as a consequence of CNT "cutting" (Figs. 4 (top, middle)), which preserves the type of conductivity. The AGNRs and ZGNRs that do not match any CNTs are all semiconducting and metallic, respectively, as summarized in Fig. 5.

Analogously, porous CNTs might have band structures similar to porous GNRs. Tight-binding calculations predicted ${ }^{28}$ that a line of SPs (separated by $\sim 12.8 \AA$ ) should cause band gap opening in ACNTs, whereas porous ZCNTs should be semiconducting regardless of the pore shape. These results are in contradiction with our $a b$ initio calculations, which show that the metallicity of pristine ACNTs (Fig. 6 a) is preserved in the porous ACNTs (Fig. 6 e), even for triangular-shape pores with clear zigzag-like rims. In metallic ZCNTs, the SP-perforation causes band gap opening, as shown in Figs. 6 (b, f), while in semiconducting ZCNTs, it causes band gap shrinking, as seen in Figs. 6 (c, g).

\section{Discussion}

The above observations allow us to build a unified model that can predict the type of conductivity in porous nanocarbons perforated with SPs and other pores that do not break the global sublattice balance. The model is based on the assumption that when NCs are perforated by a line of relatively close SPs, the type of conductivity in these PNCs is the same as in the (daughter) systems obtained from these NCs by removing all $\mathrm{C}$ atoms within a stripe going in the direction of the pores and having the same width as the pores (all dangling $\mathrm{C}$ bonds are $\mathrm{H}$-terminated). We call these modified $\mathrm{NCs}$ the daughter systems of the original NCs (two GNRs for PGNRs, one GNR for PCNTs, and a GNR-lattice for PG). This rule predicts that: (1) Porous ACNTs are metallic as the (daughter) ZGNRs; Fig. 4 (bottom) shows effective replacing of porous ACNT by pristine ZGNR. Perforating the resulting ZGNRs (and the other half of ZGNRs that can not be rolled up into CNTs) gives two metallic ZGNRs, preserving the ZGNR-metallicity. (2) Porous ZCNTs may give semiconducting or metallic AGNRs. Cutting all the AGNRs may give pairs of AGNRs with any conductivity. These results were confirmed by ab initio calculations.

We now use these rules to predict metallicity in porous AGNRs with SPs. We assume that their band gaps are $E_{B G} \simeq \min \left(E_{1 B G} ; E_{2 B G}\right)$, where $E_{1 B G}, E_{2 B G}$ are band gaps of their two daughter AGNRs (see Fig. 3 (top)). With this inference, we can derive an analytical expression describing the dependence of the band gap on the width of the porous AGNRs. For simplicity, we consider SPs positioned in the middle of AGNRs of the width of $W=a \sqrt{3}(N-1) / 2$, where $a$ is the C-C distance and $N$ is the number of dimers. By evaluating the widths of the pristine daughter AGNRs, we find that porous $\mathrm{N}$-AGNRs are potentially metallic if the number of $\mathrm{C}$-C dimers is given by at least one of these equations

$$
\begin{aligned}
& N=\left(6 k+11+(-1)^{k}\right) / 2, \quad N=6 k+3+2(-1)^{k}, \\
& N=12 k+9, \quad(k=0,1,2 \ldots) \\
& \text { i.e., if } N=5,6,7,8,9,12,14,17,18,19,20, \ldots
\end{aligned}
$$

In Fig. 7 (top), we compare the ab-initio energy band gaps in pristine ${ }^{29}, 30$ and porous AGNRs to validate the above model. In contrast to the pristine ribbons, where the metallic points emerge with the period of $3,\left(N_{m e t}=3 m+2\right)$, the band gaps of porous AGNRs have a more complex dependence. Nevertheless, the positions of the band gap minima agree with Eqns. 2.

We can extend the assumptions used in Eqns. 2 to PNCs perforated with larger and shifted honeycomb-like pores. Their presence may still be reduced to removing from the AGNRs a layer of atoms of the width given by the pore size, where the minimum band gap of the two resulting AGNRs can determine the band gap of the porous AGNR. For example, when the SP is shifted in the 11-AGNR by one honeycomb from the ribbon center, the two daughter pristine 4-AGNRs are replaced by 2-AGNR and 6-AGNR (all semiconduct-

\begin{tabular}{|c|c|c|c|c|c|c|c|c|c|c|c|c|c|c|c|c|c|c|}
\hline \multirow{4}{*}{ AGNR $<\mathbf{p}, 1>$} & dimers, $\mathbf{N}$ & 7 & 8 & 9 & 10 & 11 & 12 & 13 & 14 & \multirow{4}{*}{ ZGNR $<\mathrm{p}, \mathbf{0}>$} & 5 & 6 & 7 & 8 & 9 & 10 & 11 & 12 \\
\hline & p-index & 4 & 5 & 6 & 7 & 8 & 9 & 10 & 11 & & 4 & 5 & 6 & 7 & 8 & 9 & 10 & 11 \\
\hline & cond. & $\mathbf{s}$ & $\mathbf{m}$ & $\mathbf{s}$ & $\mathbf{s}$ & $\mathbf{m}$ & $\mathbf{s}$ & $\mathbf{s}$ & $\mathbf{m}$ & & $\mathbf{m}$ & $\mathbf{m}$ & $\mathbf{m}$ & $\mathbf{m}$ & $\mathbf{m}$ & $\mathbf{m}$ & $\mathbf{m}$ & $\mathbf{m}$ \\
\hline & geometry & $\mathrm{s}$ & $\mathrm{a}$ & $\mathrm{s}$ & $\mathrm{a}$ & $\mathrm{s}$ & $\mathrm{a}$ & $\mathrm{s}$ & $\mathrm{a}$ & & $\mathrm{a}$ & $\mathrm{s}$ & $\mathrm{a}$ & $\mathrm{s}$ & $\mathrm{a}$ & $\mathrm{s}$ & $\mathrm{a}$ & $\mathrm{s}$ \\
\hline
\end{tabular}
ing). This should lead to a band gap shrinkage, in agreement with our $a b$-initio calculations, presented in Figs. 1 (b, c). Alternatively, we can replace the SP by a double-size hexagonal pore with $24 \mathrm{C}$-atom excluded. If the 11-AGNR and 12-AGNR are perforated by such

Figure 5 Correspondence rule for GNRs and CNTs. $N$ - number of dimers forming GNR, $p$ - index for the chirality vector in GNR and CNT; cond. conductivity property (semiconducting (s) or metallic $(\mathrm{m})$ ); geometry defines the symmetry with respect to the mirror plane perpendicular to the ribbon and containing its axis: symmetric (s), asymmetric (a). Examples of AGNRs for $N=7, \ldots 14$ and ZGNRs for $N=5, \ldots 12$ are illustrated. 

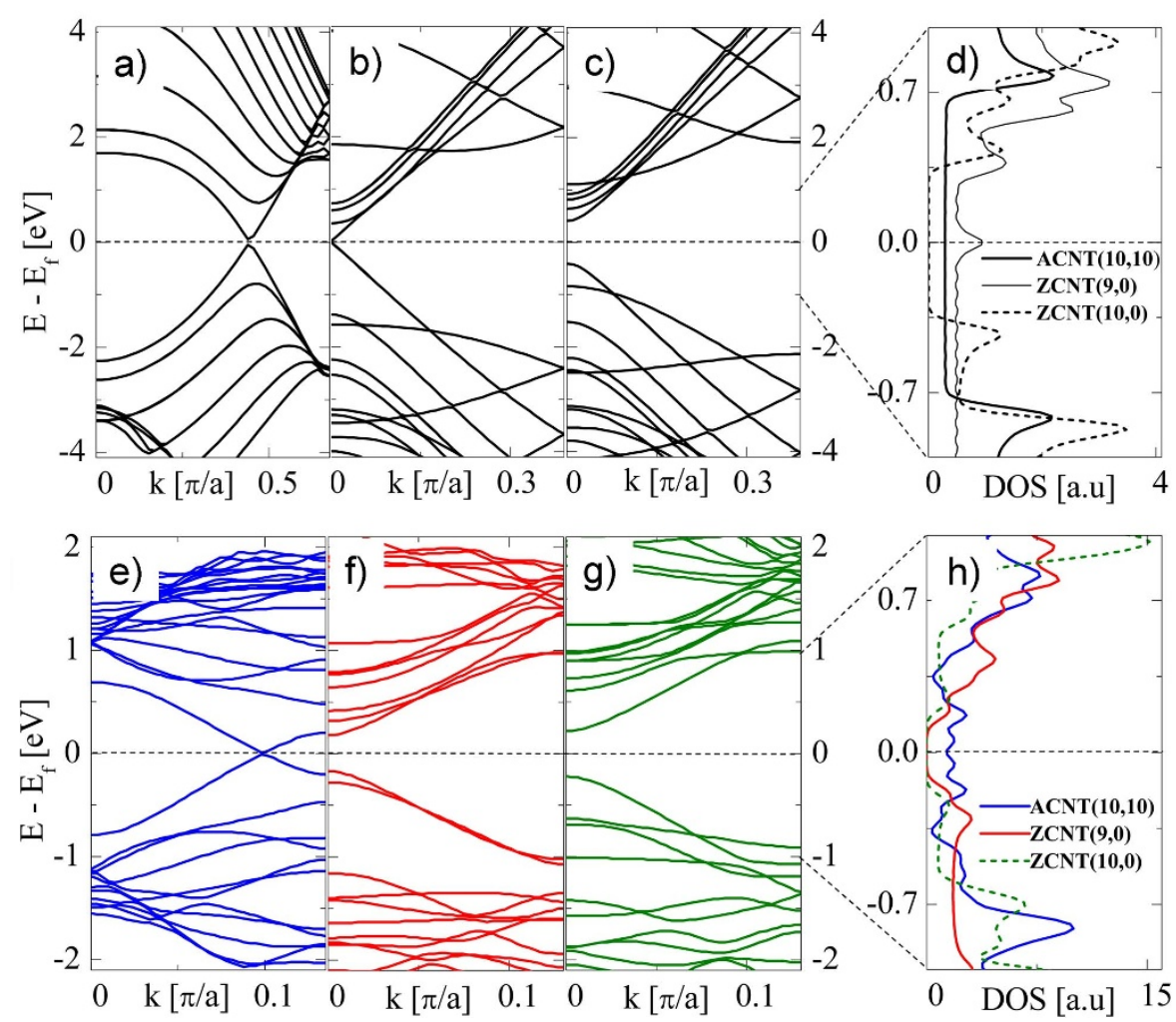

Figure 6 Band structures in pristine CNTs: a) ACNT (10,10), b) ZCNT (9,0), c) ZCNT (10,10); d) DOS. Porous CNTs: e) ACNT (10,10), f) ZCNT $(9,0), \mathrm{g}) \mathrm{ZCNT}(10,10)$; h) DOS. The energy scales for $(\mathrm{b}, \mathrm{c})$ and $(\mathrm{f}, \mathrm{g})$ cases are the same.

pores, they become semiconducting, since their cutting leads to semiconducting 2-AGNRs and 2- and 3-AGNR, respectively. These results are in agreement with ab-initio calculations, giving in 11-AGNR and 12-AGNR the band gaps of $1.1 \mathrm{eV}$ and $1.18 \mathrm{eV}$,
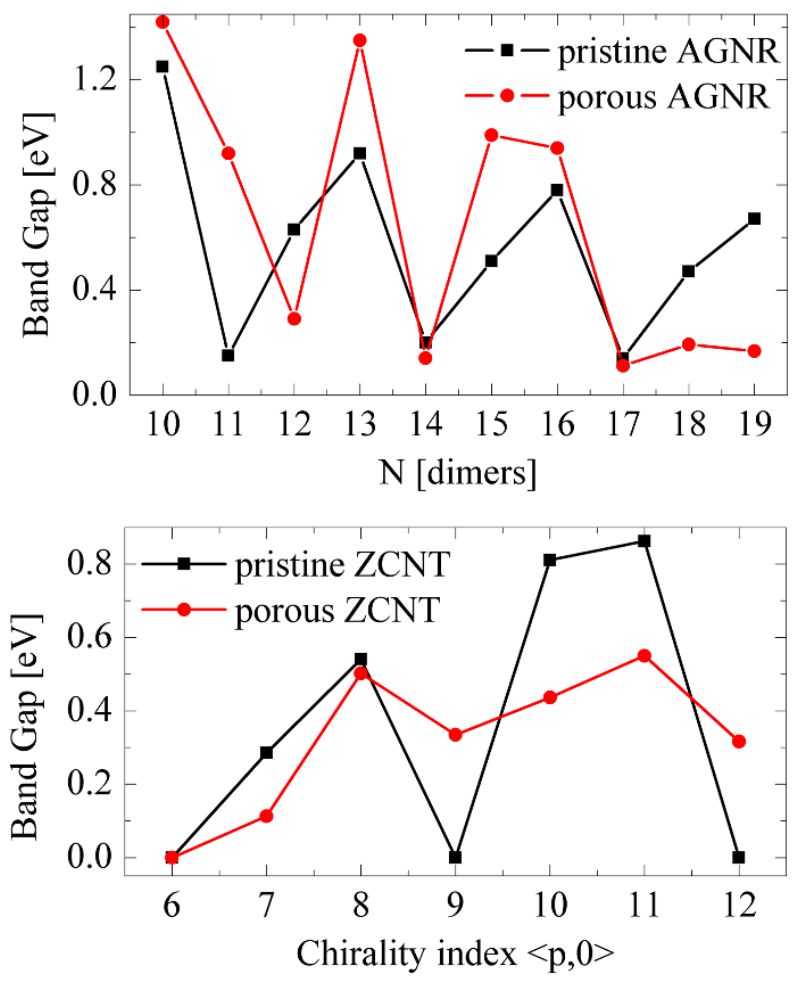

Figure $7 \mid$ (top) Dependence of the band gap in the pristine and porous AGNR on the number of dimers (the central position of the SP). (bottom) The same dependence in ZCNT on the chirality index. respectively. We have also tested the triple-size hexagonal pore (54 C-atoms excluded) in order to check how its long rims affect band structure of GNRs. Our calculations show that no additional features (e.g. flat bands at the Fermi level) appear when the GNR are perforated by these pores. In contrast, when these AGNRs are perforated with SPs in the ribbon center, only the 11-AGNR is semiconducting.

Finally, we discuss porous ZCNTs that can have any conductivity. In Fig. 7 (bottom), we present the energy band gap of porous ZCNTs in dependence on the chirality index, $p$. It exhibits similar periodicity as in the pristine ZCNTs. However, the model is not reliable in porous ZCNTs. For example, the porous $\operatorname{ZCNT}(7,0)$ and $\operatorname{ZCNT}(8,0)$ have band gaps similar to the daughter 11-AGNR and 13-AGNR, respectively. But the same is not true for the porous $\operatorname{ZCNT}(9,0)$ and ZCNT(10,0) paired with the daughter 15-AGNR and 17-AGNR, respectively. In principle, this failure might be caused by the fact that the AGNRs are not calculated deformed as the corresponding daughter $\mathrm{ZCNTs}^{31}{ }^{32}$. However, our calculations show that the bent 17AGNR has almost the same band gap as the pristine 17-AGNR. Therefore, a more quantitative approach needs to be used here.

It is of interest to see if other types of periodic modifications can also be used to tune the band structures of nanocarbons. To briefly examine this idea, we have replaced SPs by Stone-Wales 55-77 defects $^{33}$. In Fig. 8, we show the band structures of 11-AGNR, $10-Z G N R$, and graphene superlattices modified in this way. The periodic array of SW 55-77 defect in 11-AGNR leads to small bang gap opening, as shown Fig. 8 (a), in analogy to 11-AGNR with SPs (Fig.1 b). The band structure of 10-ZGNR, shown in Fig. 8 (b), is not sensitive to this perturbation, as in the SP-perturbations (Fig. $1 \mathrm{f}$ ). On the other hand, when we replace in graphene superlattices SPs with the SW 55-77 defects, we can obtain qualitatively different band structures. In particular, the band structure of graphene modified by SW 55-77 defects in the array with $N_{A}=7$ and $N_{Z}=4$ (Fig. $8 \mathrm{c}$ ) is similar to that of the SP-superlattice with the same $N_{A}$ and $N_{Z}$, but here we also observe opening of a small band gap. In Fig. 8 (d), we show the band structure of the SW-graphene superlattice $\left(N_{A}=9\right.$ 


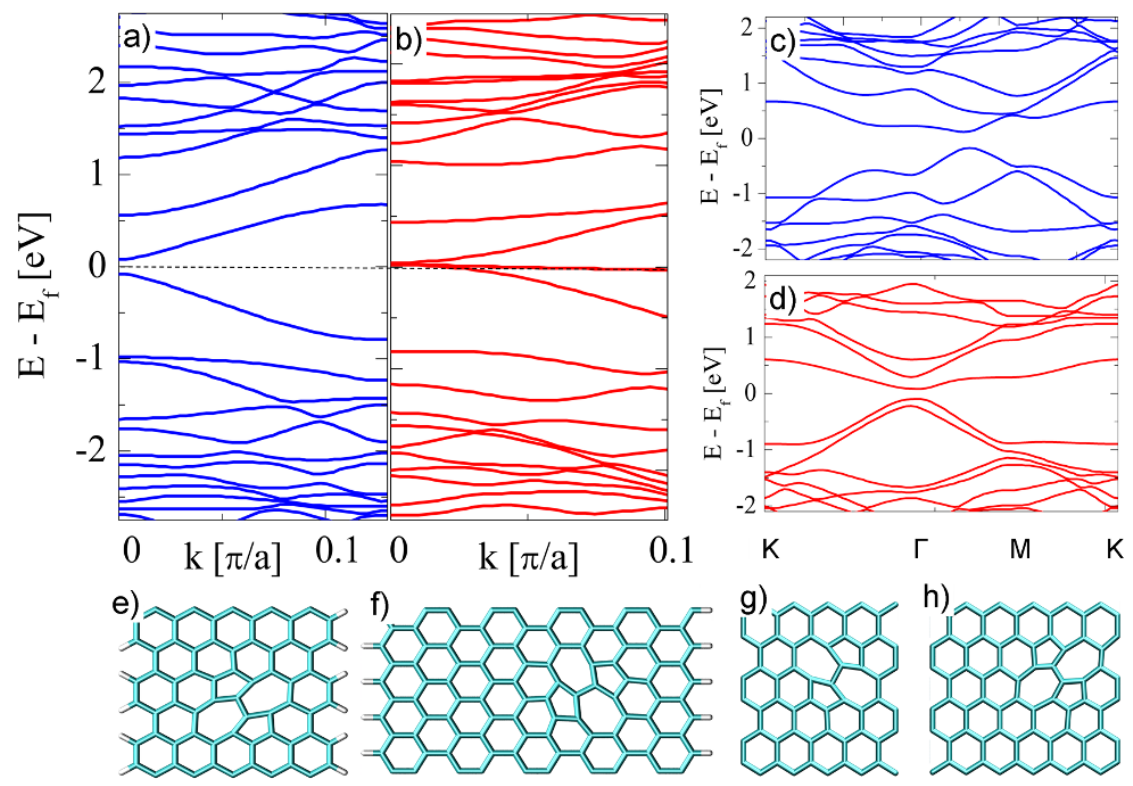

Figure $8 \mid$ Band structures of nanocarbons with periodic 55-77 Stone-Wales defects: a) 11-AGNR, b) 10-ZCNT, c) graphene superlattice with $N_{A}=7$ and $N_{Z}=4$, d) graphene superlattice with $N_{A}=9$ and $N_{Z}=4$. Figures (e-h) show unit cells for the respective cases.

and $N_{Z}=4$ ), which is semiconducting as the PG-superlattice with the same $N_{A}$ and $N_{Z}$. These observations show that periodic defects could also be used to tune band gaps in nanocarbons, but the rules might be slightly different.

For completeness, we have recalculated some of the above structures including spin polarization (we used a set of LDA and GGA functionals). It turns out, the band structures of NCs can be modified by the spin polarization (zigzag edges) [2], but the presence of SPs does not introduce additional magnetic features beyond the changes described already in the non-magnetic calculations. Interestingly, the presence of arrays of SW (55-77) defects in ZGNRs can alternate mutual orientation of the magnetic moments localized at the opposite edges, due to topological changes in the sublattices.

In summary, we have developed a unified picture of band structures in PNCs. Although, the proposed approach successfully describes band structures in many PNCs, it could be further refined to account for quantization, spin degrees of freedom, pore type, and chirality. Similar observations were made in nanocarbons perturbed by periodic SW defects. Precise knowledge of electronic structures of these materials is essential for their applications in electronics, optics, molecular sensing, and other fields.

\section{Methods}

We study the PNCs ab initio, using SIESTA 3.0-beta- $15^{34}$ in supercells of (>40 atoms), and neglect spin degrees of freedom. The length of unit cells for porous 11-AGNR and 10 -ZGNR is $12.78 \AA$ and $12.3 \AA$, respectively. The size of the supercells of PG used in our calculations varies between $12.3 \times 8.52 \AA$ (40 atoms) and $22.13 \times 21.3 \AA$ (180 atoms). We use the Perdew-Zunger LDA functional ${ }^{35}$ and pseudopotentials with the cutoff energy of $400 \mathrm{Ry}$. The calculations are done within the eigenvalue tolerance of $10^{-4} \mathrm{eV}$, using the DZP basis (double-zeta basis and polarization orbitals, 13 and 5 orbitals for $\mathrm{C}$-atom and $\mathrm{H}$-atom, respectively). The Brillouin zones of the unit cells are sampled by the Mankhorst-Pack grid ${ }^{36}$ with the spacing between the $k$-points of $\Delta k<0.01 \AA^{-1}$. Geometry optimization is carried out for all the PNCs within the conjugated gradient algorithm, until all the forces are $F<0.04 \mathrm{eV} / \AA$ and the stress in the periodic direction is $\sigma<0.01 \mathrm{GPa}$. We use a.u. for $k$ in the band structures, where only one half of the Brillouin zone is shown (symmetry).

1. Novoselov, K. S. et al. Electric field effect in atomically thin carbon films. Science 306, 666 (2004).

2. Palacios, J. J, Fernández-Rossier, J., Brey, L. Vacancy- induced magnetism in graphene and graphene ribbons. Phys. Rev. B 77, 195428 (2008).

3. Balog, R. et al. Bandgap opening in graphene induced by patterned hydrogen adsorption. Nat. Mater. 9, 315 (2010)
4. Cervantes-Sodi, F., Csányi, G., Piscanec, S., and Cervantes-Sodi, F., Csányi, G., Piscanec, S., and Ferrari, A. C. Edgefunctionalized and substitutionally doped graphene nanoribbons: electronic and spin properties. Phys. Rev. B 77, 165427 (2008)

5. Furst, J. A. et al. Electronic properties of graphene antidot lattices. New J. of Phys. 11, 095020 (2009).

6. Pedersen, T. G. et al. Graphene antidot lattices: designed defects and spin quibits. Phys. Rev. Lett. 100, 136804 (2008).

7. Vanevíc, M., Stojanovíc, M. S., and Kindermann, M. Character of electronic states in graphene antidot lattices: flat bands and spatial localization. Phys. Rev. B 80, 045410 (2009).

8. Nakada, K., Fujita, M., Dresselhaus, G., and Dresselhaus, M. Edge state in graphene ribbons: Nanometer size effect and edge shape dependence. Phys. Rev. B 54, 17954 (1996).

9. Ivanciuc, O., Bytautas, L., and Klein, D. J. Mean-field resonating-valencebond theory for unpaired $\pi$-electrons in benzenoid carbon species. J. Chem. Phys. 116, 4735 (2002).

10. Hatanaka, M. Band structures of porous graphehes. Chem. Phys. Lett. 488, 187 (2010).

11. Sint, H., Wang, B., and Král, P. Selective ion passage through functionalized graphene nanopores. J. Am. Chem. Soc. 130, 16448 (2008).

12. Jiang, D., Cooper, V. R., and Dai, S. Porous graphene as the ultimate membrane for gas separation. Nano Lett. 9, 4019 (2009).

13. Blankenburg, S. et al. Porous graphene as an atmospheric nanofilter. Small 6, 2266 (2010).

14. Bieri, M. et al. Porous graphenes: two-dimentional polymer synthesis with atomic precision. Chem. Commun. 45, 6919 (2009).

15. Postma, H. W. Ch. Rapid sequencing of individual DNA molecules in graphene nanogaps. Nano Lett. 10, 420 (2010).

16. Garaj, S. et al.Graphene as a subnanometre trans-electrode membrane. Nature 467, 190 (2010).

17. Gao, Y.-D., Kumazaki, H., Terai, J., Chida, K., and Hosoya, H. Topological factors govering the HOMO-LUMO band gap of the density of states of periodic hydrocarbon polymer networks. J. Math. Chem. 12, 279 (1993).

18. Fujita, M., Wakabayashi, K., Nakada, K., and Kusakabe, K. Peculiar localized states at zigzag graphite edges. J. Phys. Soc. Jpn. 65, 1920 (1996).

19. Shima, N. and Aoki, H. Electronic structure of superhoneycomb systems: a peculiar realization of semimetal/semiconductor classes and ferromagnetism. Phys. Rev. Lett. 71, 4389 (1993).

20. Cresti, A. et al.Charge transport in disordered graphene-based low dimentional materials. Nano Res. 1, 361 (2008).

21. Son, Y.-W., Cohen, M. L., and Louie, S. G. Energy gaps in graphene nanoribbons. Phys. Rev. Lett. 97, 216803 (2006).

22. Sols, F., Guinea, F., and Castro Neto, A. H. Coulomb Blockade in Graphene Nanoribbons. Phys. Rev. Lett. 99, 166803 (2007).

23. Lu, Y. H. et al. Effects of edge passivation by hydrogen on electronic structure of armchair graphene nanoribbons and band gap engineering. Appl. Phys. Lett. 94, 122111 (2009).

24. Boukhvalov, D. W., Katsnelson, M. I., Lichtenstein, A. I. Hydrogen on graphene: electronic structure, total energy, structural distortion and magnetism from firstprinciples calculations. Phys. Rev. B 77, 035427 (2008). 
25. We use rectangular unit cells, and calculate the band structure along the direction $K \rightarrow \Gamma \rightarrow M \rightarrow K$ in the reciprocal space, where $M=\left(k_{x}=\pi / a, k_{y}=0\right)$.

26. Fazekas, P. Lecture Notes on Electron Correlation and Magnetism (World Scientific, New York, 1999).

27. Ezawa, M. Peculiar width dependence of the electronic properties of carbon nanoribbons. Phys. Rev. B 73, 045432 (2006).

28. Sato, T., Iamde, M., and Yamabe, T. Electronic structure of porous nanotube. Synt. Met. 103, 2519 (1999).

29. Barone, V., Hod, O., and Scuseria, G. E. Electronic structure and stability of semiconducting graphene nanoribbons. Nano Lett. 6, 2748 (2006).

30. Yu, S. S., Wen, Q. B., Zheng, W. T., and Jiang, Q. Electronic properties of graphene nanoribbons with armchair-shaped edges. Mol. Sim. 34, 1085 (2008).

31. Zhang, J., Ong, K. P., and Wu, P. The influence of out-of-plane deformation on the band gap of graphene nanoribbons. J. Phys. Chem. C 114, 12749 (2010).

32. Lu, Y., Guo, J. Band gap of strained graphene nanoribbons. Nano Res. 3, 189 (2010).

33. Banhart, F., Kotakovski, J., and Krasheninnikov, A. V. Structural defects in graphene. ACS Nano. 5, 26 (2011).

34. Portal-Sánchez, D., Ordejón, P., Artacho, E., Soler, J. M. Density-functional method for very large systems with LCAO basis sets. Int. J. Quantum Chem. 65, 453 (1997).

35. Perdew, J. P., and Zunger, A. Self-interaction correction to density-functional approximations for many-electron systems. Phys. Rev. B 23, 5048 (1981).
36. Monkhorst, H. J. and Pack, J. D. Spacial points for Brillouin-zone integrations. Phys. Rev. B 13, 5188 (1976).

\section{Acknowledgments}

We acknowledge support from the NSF CBET-0932818 grant. The calculations were performed at the NERSC, NCSA, and CNM supercomputers. We thank Niladri Patra, Irena Yzeiri, and Cathy Skontos for technical help.

\section{Authors contributions}

A.B. and P.K. wrote the main manuscript text. A.B. performed all the calculations. All authors discussed the results and commented on the manuscript.

\section{Additional information}

Competing financial interests: The authors declare no competing financial interests.

License: This work is licensed under a Creative Commons

Attribution-NonCommercial-ShareAlike 3.0 Unported License. To view a copy of this license, visit http://creativecommons.org/licenses/by-nc-sa/3.0/

How to cite this article: Baskin, A. \& Král, P. Electronic structures of porous nanocarbons. Sci. Rep. 1, 36; DOI:10.1038/srep00036 (2011). 